\title{
LIMIT THEOREMS FOR THE PAINTING OF GRAPHS BY CLUSTERS
}

\author{
OLIVIER GARet ${ }^{1}$
}

\begin{abstract}
We consider a generalization of the so-called divide and color model recently introduced by Häggström. We investigate the behavior of the magnetization in large boxes of the lattice $\mathbb{Z}^{d}$ and its fluctuations. Thus, Laws of Large Numbers and Central Limit Theorems are proved, both quenched and annealed. We show that the properties of the underlying percolation process deeply influence the behavior of the coloring model. In the subcritical case, the limit magnetization is deterministic and the Central Limit Theorem admits a Gaussian limit. Conversely, the limit magnetization is not deterministic in the supercritical case and the limit of the Central Limit Theorem is not Gaussian, except in the particular model with exactly two colors which are equally probable. We also prove a Central Limit Theorem for the size of the intersection of the infinite cluster with large boxes in supercritical bond percolation.
\end{abstract}

Mathematics Subject Classification. 60K35, 82B20, 82B43.

Received May 15, 2001. Revised July 2 and October 3, 2001.

\section{INTRODUCTION}

The aim of this paper is to give some results concerning a pretty and natural model for the dependent coloring of vertices of a graph. This model has recently been introduced by Häggström [7], who presented the first results, especially concerning the presence (or absence) of percolation and the quasilocality properties. The model is easily described: choose a graph at random according to bond percolation, and then paint randomly and independently the different clusters, each cluster being monochromatic. There are several motivations for the study of such a model, the most relevant being its links with Ising or Potts models. We refer to the examples of the present article and to the introduction of Häggström's paper for detailed motivations.

In Häggström's model, the panel consisted of a finite number of colors, which were chosen according to a measure $\nu$ on $\mathbb{R}$ with finite support. For our purpose, the natural assumptions will only be the existence of a first or a second moment for $\nu$.

We will study the mean magnetization of the random field $(X(x))_{x \in \mathbb{Z}^{d}}$ in large boxes $\Lambda_{n}=\{-n, \ldots, n\}^{d}$ : we will identify the limit

$$
M=\lim _{n \rightarrow \infty} \frac{1}{\left|\Lambda_{n}\right|} \sum_{x \in \Lambda_{n}} X(x)
$$

\footnotetext{
Keywords and phrases: Percolation, coloring model, Law of Large Number, Central Limit Theorem.

${ }^{1}$ Laboratoire de Mathématiques, Applications et Physique Mathématique d'Orléans, UMR 6628, Université d'Orléans, BP. 6759, 45067 Orléans Cedex 2, France; e-mail: Olivier.Garet@labomath.univ-orleans.fr 
and determinate its variations: we will prove Central Limit Theorems for quantities such as

$$
\frac{1}{\left(\left|\Lambda_{n}\right|\right)^{1 / 2}}\left(\left(\sum_{x \in \Lambda_{n}} X(x)\right)-\left|\Lambda_{n}\right| M\right) .
$$

There are several natural questions: when is $M$ deterministic? What is the influence of the underlying bond percolation? When is there convergence to a normal law in the Central Limit Theorem?

These questions can be asked in two different approaches. Here we shall use the vocabulary usually used in the theory of random media.

- The quenched point of view: Limit Theorems are formulated once the graph has been (randomly) fixed.

- The annealed point of view: Limit Theorems are formulated under the randomization of the graph. We average over the possible issues of the bond percolation process.

Indeed, we will show that the properties of the underlying percolation process deeply influence the behavior of the coloring model. In the subcritical case, the limit magnetization is deterministic and the Central Limit Theorem admits a Gaussian limit. Conversely, the limit magnetization is not deterministic in the supercritical case and the limit of the Central Limit Theorem is not Gaussian, except in the particular model with exactly two colors which are equally probable. As examples, we will study the case where $\nu$ is " $+/-$ " valued and the case where $\nu$ is a Gaussian measure.

\section{Notations}

We will deal here with stochastic processes indexed by $\mathbb{Z}^{d}$. Their definition will be related to some subgraphs of the $d$-dimensional cubic lattice $\mathbb{L}^{d}$, which is defined by $\mathbb{L}^{d}=\left(\mathbb{Z}^{d}, \mathbb{E}^{d}\right)$, where $\mathbb{E}^{d}=\left\{\{x, y\} \subset \mathbb{Z}^{d} ; \sum_{i=1}^{d}\left|x_{i}-y_{i}\right|\right.$ $=1\}$. In the following, the expression "subgraph of $\mathbb{L}^{d "}$ will always be employed for each graph of the form $G=\left(\mathbb{Z}^{d}, E\right)$ where $E$ is a subset of $\mathbb{E}^{d}$. We denote by $\mathcal{S}\left(\mathbb{L}^{d}\right)$ the set of all subgraphs of $\mathbb{L}^{d}$. We say that two vertices $x, y \in \mathbb{Z}^{d}$ are adjacent in $G$ if $\{x, y\} \in E$. Two vertices $x, y \in \mathbb{Z}^{d}$ are said to be connected in $G$ if one can find a sequence of vertices containing $x$ and $y$ such that each element of the sequence is adjacent in $G$ with the next one. A subset $C$ of $\mathbb{Z}^{d}$ is said to be connected if each pair of vertices in $C$ is connected. The maximal connected sets are called the connected components. They partition $\mathbb{Z}^{d}$. The connected component of $x$ is denoted by $C(x)$. The connected components are also called clusters. A subset $D$ of $\mathbb{Z}^{d}$ is said to be independent if no pair in $D$ is constituted by adjacent vertices.

Here we will consider subgraphs of $\mathbb{L}^{d}$ which are generated by Bernoulli bond percolation on $\mathbb{L}^{d}$. Thus, we will denote by $\mu_{p}$ the image measure of $\left(\{0,1\}^{\mathbb{E}^{d}}, \mathcal{B}\left(\{0,1\}^{\mathbb{E}^{d}}\right),\left((1-p) \delta_{0}+p \delta_{1}\right)^{\otimes \mathbb{E}^{d}}\right)$ by

$$
x \mapsto\left(\mathbb{Z}^{d},\left\{e \in \mathbb{E}^{d} ; x_{e}=1\right\}\right),
$$

where $p \in(0,1)$.

Let us choose a graph $G$ at random under $\mu_{p}$ and recall the definition of some basic objects in percolation theory (for more details, see for example Grimmett [4]).

- The probability that 0 belongs to an infinite cluster:

$\theta(p)=\mu_{p}(|C(0)|=+\infty)$.

- The critical probability:

$p_{c}=\inf \{p \in(0,1) ; \theta(p)>0\}$.

- The mean size of a finite cluster:

$\chi^{f}(p)=\sum_{k=1}^{+\infty} k \mu_{p}(|C(0)|=k)$.

The following results will be frequently used:

- $\mu_{p}$ is translation-invariant. As it is isomorphic to $\left(\{0,1\}^{\mathbb{E}^{d}}, \mathcal{B}\left(\{0,1\}^{\mathbb{E}^{d}}\right),\left((1-p) \delta_{0}+p \delta_{1}\right)^{\otimes \mathbb{E}^{d}}\right)$, its tail $\sigma$-field is trivial and the ergodic theorem can be employed with full power. 
- If $p \in\left(0, p_{c}\right)$, then $G$ contains no infinite cluster.

- If $p \in\left(p_{c}, 1\right)$, then $G$ contains $\mu_{p}$-almost surely one unique infinite cluster.

- If $p \neq p_{c}$, then $\chi^{f}(p)<+\infty$.

If $G$ is a subgraph of $\mathbb{L}^{d}$ and if $\nu$ is a probability measure on $\mathbb{R}$, we will define the color-probability $P^{G, \nu}$ as follows: $P^{G, \nu}$ is the unique measure on $\left(\mathbb{R}^{\mathbb{Z}^{d}}, \mathcal{B}\left(\mathbb{R}^{\mathbb{Z}^{d}}\right)\right)$ under which the canonical projections $X_{i}$ - defined, as usual by $X_{i}(\omega)=\omega_{i}$ - satisfy

- for each $i \in \mathbb{Z}^{d}$, the law of $X_{i}$ is $\nu$;

- for each independent set $S \subset \mathbb{Z}^{d}$, the variables $\left(X_{i}\right)_{i \in S}$ are independent;

- for each connected set $S \subset \mathbb{Z}^{d}$, the variables $\left(X_{i}\right)_{i \in S}$ are identical.

The randomized color-measure is defined by

$$
P^{p, \nu}=\int_{\mathcal{S}\left(\mathbb{L}^{d}\right)} P^{G, \nu} \mathrm{d} \mu_{p}(G) .
$$

We also note $\Lambda_{n}=\{-n, \ldots, n\}^{d}$ and define the tail $\sigma$-field $\mathcal{T}$ by

$$
\mathcal{T}=\underset{n \geq 1}{\bigcap} \mathcal{F}_{\mathbb{Z}^{d} \backslash \Lambda_{n}}
$$

where $\mathcal{F}_{S}$ is the $\sigma$-field generated by the $\left(X_{i}\right)_{i \in S}$.

\section{LAWS OF LARGE NUMBERS}

\subsection{Annealed law of large numbers}

Theorem 2.1. Let $\nu$ be a probability measure on $\mathbb{R}$ with a first moment. We put $m=\int_{\mathbb{R}} x \mathrm{~d} \nu(x)$.

Then, for $p \in(0,1) \backslash\left\{p_{c}\right\}$ :

$$
\lim _{n \rightarrow+\infty} \frac{1}{\left|\Lambda_{n}\right|} \sum_{x \in \Lambda_{n}} X(x)=(1-\theta(p)) m+\theta(p) Z \quad P^{p, \nu} \text {-almost surely }
$$

where $Z$ is the value taken by $X()$ along the infinite component if it exists, and 0 else.

Proof. We will build here $P^{p, \nu}$ as the image of a product measure by an appropriate transform.

Let $\Omega=\{0,1\}^{\mathbb{E}^{d}} \times \mathbb{R}^{\mathbb{Z}^{d}}$ and consider the probability measure $P=\left((1-p) \delta_{0}+p \delta_{1}\right)^{\otimes \mathbb{E}^{d}} \otimes \nu^{\otimes \mathbb{Z}^{d}}$ on $(\Omega, \mathcal{B}(\Omega))$.

We define $G((\eta, \omega))$ as the subgraph of $\mathbb{L}^{d}$ which is such that for each $i \in \mathbb{E}^{d}$, the vertex $i$ is open if and only if $\eta_{i}=1$. For $x \in \mathbb{Z}^{d}, C(x)((\eta, \omega))$ is the cluster of $x$ in this graph. We also define the random set $I=\left\{x \in \mathbb{Z}^{d} ;|C(x)|=+\infty\right\}$. For each subset $A \subset \mathbb{Z}^{d}$, we define inf $A$ as the smallest edge of $A$ in the lexical ordering.

We can now define the random field $(X(k))_{k \in \mathbb{Z}^{d}}$ by $X(k)((\eta, \omega))=\omega_{\text {inf } C(k)(\eta, \omega)}$. It is easy to see that $P_{X}=P^{p, \nu} . Z$ is defined by $Z((\eta, \omega))=0$ if $I(\eta, \omega)=\varnothing$ and $Z(\eta, \omega)=\omega_{\inf I(\eta, \omega)}$ else.

Let us define

$$
Q_{n}=\frac{1}{\left|\Lambda_{n}\right|} \sum_{x \in \Lambda_{n}} X(x)
$$

Since $P^{p, \nu}$ is translation-invariant, the ergodic Theorem ensures that $Q_{n}$ would $P^{p, \nu}$-almost surely and in $\mathrm{L}^{1}\left(P^{p, \nu}\right)$ converge. Our goal is now to identify this limit.

Since $G$ has almost surely at most one infinite cluster, we have

$$
Q_{n}=D_{n}+\frac{\left|I \cap \Lambda_{n}\right|}{\left|\Lambda_{n}\right|} Z \text { a.s., }
$$


where

$$
D_{n}=\frac{1}{\left|\Lambda_{n}\right|} \sum_{x \in \Lambda_{n}} X^{\prime}(x) \text { with } X^{\prime}(x)=X(x) \mathbb{1}_{|C(x)|<+\infty}
$$

Since $\left|\Lambda_{n} \cap I\right|=\sum_{x \in \Lambda_{n}} \mathbb{1}_{\{|C(x)|=+\infty\}}$, it follows from the ergodic theorem that

$$
\lim _{n \rightarrow+\infty} \frac{1}{\left|\Lambda_{n}\right|}\left|\Lambda_{n} \cap I\right|=\mathbb{E} \mathbb{1}_{\{|C(0)|=+\infty\}}=\theta(p) \text { a.s. and in } \mathrm{L}^{1} .
$$

It remains to identify the limit of $\left(D_{n}\right)_{n \geq 1}$.

For each finite set $\Lambda \subset \mathbb{Z}^{d}$, let us denote by $\mathcal{F}_{\Lambda}^{\prime}$ the $\sigma$-field generated by the independent variables indexed by edges and vertices which stay in the outside of $\Lambda$. Precisely, $\mathcal{F}_{\Lambda}^{\prime}=\sigma\left((\eta, \omega) \mapsto\left(\eta_{e}, \omega_{k}\right) ; e \cap \Lambda=\varnothing\right.$ and $\left.k \notin \Lambda\right)$.

For each $(\eta, \omega)$, we denote by $\left(\tilde{\eta}_{\Lambda}, \omega_{\Lambda}^{\prime}\right)$ the configuration obtained from $(\eta, \omega)$ as follows: one removes the vertices which intersect $\Lambda$ and replaces the previous color of the edges inside $\Lambda$ by the color 0 . The colors in the outside of $\Lambda$ remain unchanged. Precisely, $\left(\omega_{\Lambda}^{\prime}\right)_{k}=\omega_{k} \mathbb{1}_{k \notin \Lambda}$ for each $k \in \mathbb{Z}^{d}$ and $\left(\tilde{\eta}_{\Lambda}\right)_{e}=\eta_{e} \mathbb{1}_{e \cap \Lambda=\varnothing}$ for each $e \in \mathbb{E}^{d}$.

Since $X^{\prime}\left(\left(\omega_{\Lambda}^{\prime}, \tilde{\eta}_{\Lambda}\right)\right)$ only differs from $X^{\prime}((\omega, \eta))$ in the color of a finite number of edges, it follows that

$\lim _{n \rightarrow+\infty} D_{n}((\omega, \eta))=\lim _{n \rightarrow+\infty} D_{n}\left(\left(\omega_{\Lambda}^{\prime}, \tilde{\eta}_{\Lambda}\right)\right)$. But for each $n,(\omega, \eta) \mapsto D_{n}\left(\left(\omega_{\Lambda}^{\prime}, \tilde{\eta}_{\Lambda}\right)\right)$ is $\mathcal{F}_{\Lambda}^{\prime}$-measurable. Then $(\omega, \eta) \mapsto \lim _{n \rightarrow+\infty} D_{n}((\omega, \eta))$ is $\mathcal{F}_{\Lambda}^{\prime}$-measurable. Since this holds for each finite $\Lambda,(\omega, \eta) \mapsto \quad \lim _{n \rightarrow+\infty} D_{n}((\omega, \eta))$ is measurable with respect to the tail $\sigma$-field. Then, it is constant by Kolmogorov's $0-1$ law.

Since $\mathbb{E} D_{n}=(1-\theta(p)) m$ for each $n$, this constant is necessarily $(1-\theta(p)) m$.

This concludes the proof.

We will now formulate an easy, but important corollary:

Corollary 2.2. $\quad$ - $Z$ is $\mathcal{T}$-measurable.

- For $p>p_{c}, \mathcal{T}$ is not trivial under $P^{p, \nu}$ as soon as $\nu$ is not a Dirac measure.

Proof. The first point is a consequence of the formula given in Theorem 2.1 and the second point is a consequence of the first one, because $Z$ is non constant as soon as $\nu$ is not a Dirac measure.

The fact that $Z$ is $\mathcal{T}$-measurable is important for the formulation of annealed results, because the environment is forgotten once we have randomized under $\mu_{p}$. Indeed, despite the infinite component can not always be recovered, the value of $X()$ along this component can.

\subsection{Quenched law of large numbers}

Theorem 2.3. Let $\nu$ be a probability measure on $\mathbb{R}$ with a first moment. We put $m=\int_{\mathbb{R}} x \mathrm{~d} \nu(x)$. Let $p \in(0,1) \backslash\left\{p_{c}\right\}$.

For $\mu_{p}$-almost all $G$, the following holds:

$$
\lim _{n \rightarrow+\infty} \frac{1}{\left|\Lambda_{n}\right|} \sum_{x \in \Lambda_{n}} X(x)=(1-\theta(p)) m+\theta(p) Z \quad P^{G, \nu} \text {-almost surely }
$$

where $Z$ is the value taken by $X()$ along the infinite component if it exists, and 0 else. 
Proof. Let $C=\left\{\lim _{n \rightarrow+\infty} \frac{1}{\left|\Lambda_{n}\right|} \sum_{x \in \Lambda_{n}} X(x)=(1-\theta(p)) m+\theta(p) Z\right\}$. We have

$$
0=1-P^{p, \nu}(C)=\int_{\mathcal{S}\left(\mathbb{L}^{d}\right)}\left(1-P^{G, \nu}(C)\right) \mathrm{d} \mu_{p}(G) .
$$

Since $\left(1-P^{G, \nu}(C)\right) \geq 0$, it follows that $1-P^{G, \nu}(C)=0$ for $\mu_{p^{-}}$almost all $G$.

\subsection{Examples}

\subsection{1. "+/-" valued spin system}

It is the simplest model that we can study, since it only takes two values: "+1" and " -1 ", with probability $\alpha$ and $1-\alpha$. In the terminology of Häggström [5], it is denoted as the $r+s$-state fractional fuzzy Potts model at inverse temperature $-\frac{1}{2} \ln (1-p)$, with $r=\alpha$ and $s=1-\alpha$. This name alludes to the fact that the fuzzy Potts model can be realized using random clusters by an analogous painting procedure. For more details, see Häggström [5].

We point out that for $\mu_{p}$-almost all $G$, we have

$$
P^{G,(1-\alpha) \delta_{-1}+\alpha \delta_{+1}}=\alpha \lim _{\beta \rightarrow+\infty} \mathcal{I}_{G, \beta, h}^{+}+(1-\alpha) \lim _{\beta \rightarrow+\infty} \mathcal{I}_{G, \beta, h}^{-}
$$

where $\lim _{\beta \rightarrow+\infty} \mathcal{I}_{G, \beta, h}^{+}$

(resp. $\left.\lim _{\beta \rightarrow+\infty} \mathcal{I}_{G, \beta, h}^{-}\right)$is the Ising Gibbs measure on $G$ at inverse temperature $\beta$ with the external field $h=\frac{1}{2} \ln (\alpha /(1-\alpha))$ which is maximal (resp. minimal) for the stochastic domination. Thus,

$$
P^{p,(1-\alpha) \delta_{-1}+\alpha \delta_{+1}}=\lim _{\beta \rightarrow+\infty} \int_{\mathcal{S}\left(\mathbb{L}^{d}\right)} \alpha \mathcal{I}_{G, \beta, h}^{+}+(1-\alpha) \mathcal{I}_{G, \beta, h}^{-} \mathrm{d} \mu_{p}(G)
$$

In this sense, we can say that $P^{p,(1-\alpha) \delta_{-1}+\alpha \delta_{+1}}$ arises at the zero temperature limit of an Ising model on a randomly diluted lattice. For precise definitions and results relative to Ising ferromagnets on random subgraphs generated by bond percolation, see Georgii [3] and as well the recent article of Häggström et al. [6].

If we choose $\nu=(1-\alpha) \delta_{-1}+\alpha \delta_{1}$ with $\alpha \in(0,1)$, it follows that the magnetization is

$$
M=\lim _{n \rightarrow+\infty} \frac{1}{\left|\Lambda_{n}\right|} \sum_{x \in \Lambda_{n}} X(x)= \begin{cases}2 \alpha(1-\theta(p))+2 \theta(p)-1 & \text { with probability } \alpha \\ 2 \alpha(1-\theta(p))-1 & \text { with probability } 1-\alpha .\end{cases}
$$

When $p \in\left(0, p_{c}\right)$, the magnetization is deterministic. Moreover, it follows from (3) that the sign of the magnetization is deterministic if and only if

$$
\max (\alpha, 1-\alpha)(1-\theta(p)) \geq \frac{1}{2}
$$

Note that if $\theta(p) \geq \frac{1}{2}$ the sign of the magnetization can not be deterministic.

In the case $\alpha=\frac{1}{2}$, the annealed law of the magnetization has been identified by Häggström ([7], Prop. 2.1) using a spin-flip argument. 


\subsubsection{A quenched Gaussian system}

Here we choose $\nu=\mathcal{N}(0,1)$. For each $G, P^{G, \nu}$ is a Gaussian measure. Here, we have

$$
M=\lim _{n \rightarrow+\infty} \frac{1}{\left|\Lambda_{n}\right|} \sum_{x \in \Lambda_{n}} X(x)=\theta(p) Z
$$

In other words, $M$ is almost surely null when $p<p_{c}$ and $M \sim \mathcal{N}\left(0, \theta(p)^{2}\right)$ when $p>p_{c}$.

We emphasize that these Large Numbers Theorems are valid both quenched and annealed. This will no longer be so simple for Central Limit Theorems.

\section{Central Limit Theorems}

In the following, we write $X_{n} \Longrightarrow \mu$ to denote the weak convergence of a sequence of random variables $\left(X_{n}\right)_{n \geq 1}$ to a probability measure $\mu$.

\subsection{Quenched Central Limit Theorem}

Theorem 3.1. Let $\nu$ be a probability measure on $\mathbb{R}$ with a second moment. We put $m=\int_{\mathbb{R}} x \mathrm{~d} \nu(x)$ and $\sigma^{2}=\int_{\mathbb{R}}(x-m)^{2} \mathrm{~d} \nu(x)$.

For $\mu_{p}$-almost all $G$, the following holds:

- The subcritical case

If $p \in\left(0, p_{c}\right)$, then

$$
\frac{1}{\left|\Lambda_{n}\right|^{1 / 2}}\left(\sum_{x \in \Lambda_{n}}(X(x)-m)\right) \Longrightarrow \mathcal{N}\left(0, \chi^{f}(p) \sigma^{2}\right) .
$$

- The supercritical case

If $p \in\left(p_{c}, 1\right)$, then

$$
\frac{1}{\left|\Lambda_{n}\right|^{1 / 2}}\left(\sum_{x \in \Lambda_{n} \backslash I}(X(x)-m)\right) \Longrightarrow \mathcal{N}\left(0, \chi^{f}(p) \sigma^{2}\right)
$$

where I is the infinite component of $G$.

The following lemma will be very useful:

Lemma 3.2. For each subgraph $G$ of $\mathbb{L}^{d}$, let us denote by $\left(A_{i}\right)_{i \in I}$ the partition of $G$ into connected components.

Then, if $p \neq p_{c}$, we have for $\mu_{p}$-almost all $G$ :

$$
\lim _{n \rightarrow \infty} \frac{1}{\left|\Lambda_{n}\right|} \sum_{i \in I ;\left|A_{i}\right|<+\infty}\left|A_{i} \cap \Lambda_{n}\right|^{2}=\chi^{f}(p)
$$

where

$$
\chi^{f}(p)=\sum_{k=1}^{+\infty} k P(|C(0)|=k) .
$$


Proof. Let us define $C^{\prime}(x)$ by

$$
C^{\prime}(x)= \begin{cases}C(x) & \text { if }|C(x)|<+\infty \\ \varnothing & \text { otherwise }\end{cases}
$$

and $C_{n}^{\prime}(x)=C^{\prime}(x) \cap \Lambda_{n}$.

It is easy to see that

$$
\sum_{i \in I ;\left|A_{i}\right|<+\infty}\left|A_{i} \cap \Lambda_{n}\right|^{2}=\sum_{x \in \Lambda_{n}}\left|C_{n}^{\prime}(x)\right|
$$

We have $\left|C_{n}^{\prime}(x)\right| \leq|C(x)|$, and the equality holds if and only if $C^{\prime}(x) \subset \Lambda_{n}$.

The quantity residing in connected components intersecting the boundary of $\Lambda_{n}$ can be controlled using well-known results about the distribution of the size of finite clusters. In both subcritical case and supercritical case, we can found $K>0$ and $\beta>0$ such that

$$
P(+\infty>|C(x)| \geq n) \leq \exp \left(-K n^{\beta}\right) .
$$

(We can take $\beta=1$ when $p<p_{c}$ and $\beta=(d-1) / d$ if $p>p_{c}$. See for example the reference book of Grimmett [4] for a detailed historical bibliography.) It follows from a standard Borel-Cantelli argument that for $\mu_{p}$-almost all $G$, there exists a (random) $N$ such that

$$
\forall n \geq N \quad \max _{x \in \Lambda_{n}}\left|C^{\prime}(x)\right| \leq(\ln n)^{2 / \beta} .
$$

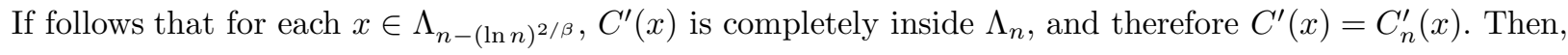

$$
\begin{aligned}
\sum_{x \in \Lambda_{n-(\ln n)^{2 / \beta}}\left|C^{\prime}(x)\right|} \leq \sum_{x \in \Lambda_{n}}\left|C_{n}^{\prime}(x)\right| \leq \sum_{x \in \Lambda_{n}}\left|C^{\prime}(x)\right| \\
\frac{1}{\left|\Lambda_{n}\right|} \sum_{x \in \Lambda_{n-(\ln n)^{2 / \beta}}\left|C^{\prime}(x)\right|} \leq \frac{1}{\left|\Lambda_{n}\right|} \sum_{x \in \Lambda_{n}}\left|C_{n}^{\prime}(x)\right| \leq \frac{1}{\left|\Lambda_{n}\right|} \sum_{x \in \Lambda_{n}}\left|C^{\prime}(x)\right| .
\end{aligned}
$$

By the ergodic Theorem, we have $\mu_{p}$-almost surely

$$
\lim _{n \rightarrow+\infty} \frac{1}{\left|\Lambda_{n}\right|} \sum_{x \in \Lambda_{n}}\left|C^{\prime}(x)\right|=\mathbb{E}\left|C^{\prime}(0)\right|=\chi^{f}(p) .
$$

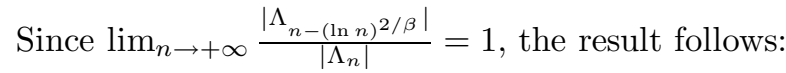

Remark. If we forget technical controls, the key point of this proof is the identity (5). It is interesting to note that Grimmett [4] used an analogous trick to prove that $\lim _{n \rightarrow+\infty} k(n) /\left|\Lambda_{n}\right|=\kappa(p)$-almost surely, where $k(n)$ is the number of open clusters in $\Lambda_{n}$ and $\kappa(p)=\mathbb{E}|C(0)|^{-1}$.

We can now proof Theorem 3.1. For simplicity, we will give the proof in the supercritical case - which contains the proof of the subcritical case.

Proof.

$$
\sum_{x \in \Lambda_{n} \backslash I}(X(x)-m)=\sum_{i=1}^{+\infty}\left|C_{n}^{\prime}\left(a_{i}\right)\right|\left(X\left(a_{i}\right)-m\right) .
$$


Then

$$
\frac{1}{\left|\Lambda_{n}\right|^{1 / 2}} \sum_{x \in \Lambda_{n} \backslash I}(X(x)-m)=\left(\frac{s_{n}^{2}}{\left|\Lambda_{n}\right|}\right)^{1 / 2} \frac{1}{s_{n}} \sum_{i=1}^{+\infty}\left|C_{n}^{\prime}\left(a_{i}\right)\right|\left(X\left(a_{i}\right)-m\right),
$$

with

$$
s_{n}^{2}=\sum_{i=1}^{+\infty}\left|C_{n}^{\prime}\left(a_{i}\right)\right|^{2}
$$

By Lemma 3.2, we have for $\mu^{p}$-almost all $G \lim _{n \rightarrow+\infty} \frac{s_{n}^{2}}{\left|\Lambda_{n}\right|}=\chi^{f}(p)$.

Now, it remains to prove that

$$
\frac{1}{s_{n}} \sum_{i=1}^{+\infty}\left|C_{n}^{\prime}\left(a_{i}\right)\right|\left(X\left(a_{i}\right)-m\right) \Longrightarrow \mathcal{N}\left(0, \sigma^{2}\right)
$$

Therefore, we will prove that for $\mu^{p}$-almost all $G$, the sequence $Y_{n, k}=\left|C_{n}^{\prime}\left(a_{i}\right)\right|\left(X\left(a_{i}\right)-m\right)$ satisfies the Lindeberg condition. For each $\varepsilon>0$, we have

$$
\begin{aligned}
\sum_{k=1}^{+\infty} \frac{1}{s_{n}^{2}} \int_{\left|Y_{n, k}\right| \geq \varepsilon s_{n}} Y_{n, k}^{2} \mathrm{~d} P^{G, \nu} & =\sum_{k=1}^{+\infty} \frac{\left|C_{n}^{\prime}\left(a_{k}\right)\right|^{2}}{s_{n}^{2}} \int_{\left|C_{n}^{\prime}\left(a_{k}\right)\right||x| \geq \varepsilon s_{n}}(x-m)^{2} \mathrm{~d} \nu(x) \\
& \leq \int_{|x| \geq \frac{\varepsilon}{\eta_{n}}}(x-m)^{2} \mathrm{~d} \nu(x),
\end{aligned}
$$

with $\eta_{n}=\frac{\sup _{k \geq 1}\left|C_{n}^{\prime}\left(a_{k}\right)\right|}{s_{n}}$. Thus, the Lindeberg condition is fulfilled if $\lim \eta_{n}=0$. But we have already seen that $s_{n} \sim\left(\chi^{f}(p)\left|\Lambda_{n}\right|\right)^{1 / 2}$, whereas $\sup _{k \geq 1}\left|C_{n}^{\prime}\left(a_{k}\right)\right|=O\left((\ln n)^{2 / \beta}\right)$. This concludes the proof.

\subsection{Annealed Central Limit Theorem}

Theorem 3.3. Let $\nu$ be a probability measure on $\mathbb{R}$ with a second moment. We put $m=\int_{\mathbb{R}} x \mathrm{~d} \nu(x)$ and $\sigma^{2}=\int_{\mathbb{R}}(x-m)^{2} \mathrm{~d} \nu(x)$. Let $p \in(0,1) \backslash\left\{p_{c}\right\}$. We emphasize that $G$ is randomized under $\mu_{p}$.

- The subcritical case

If $p \in\left(0, p_{c}\right)$, then

$$
\frac{1}{\left|\Lambda_{n}\right|^{1 / 2}}\left(\sum_{x \in \Lambda_{n}}(X(x)-m)\right) \Longrightarrow \mathcal{N}\left(0, \chi^{f}(p) \sigma^{2}\right)
$$

- The supercritical case

If $p \in\left(p_{c}, 1\right)$, then

$$
\left.\frac{1}{\left|\Lambda_{n}\right|^{1 / 2}}\left(\sum_{x \in \Lambda_{n}} X(x)-((1-\theta(p)) m+\theta(p) Z)\left|\Lambda_{n}\right|\right)\right) \Longrightarrow \gamma
$$


where $\gamma$ is the image of $\mathcal{N}\left(0, \chi^{f}(p) \sigma^{2}\right) \times \mathcal{N}\left(0, \sigma_{p}^{2}\right) \times \nu$ by $(x, y, z) \mapsto x+y(z-m)$, with

$$
\sigma_{p}^{2}=\sum_{k \in \mathbb{Z}^{d}}\left(P(0 \in \text { Iand } k \in I)-\theta(p)^{2}\right)
$$

where $I$ is the infinite component of $G$.

In the subcritical case, the Annealed Central Limit Theorem is a simple consequence of the Quenched Central Limit Theorem.

In order to prove this result in the supercritical case, we will need a Central Limit Theorem related to the variations of the size of the intersection of the infinite cluster with large boxes.

Yu Zhang had recently proved the following [12]:

$$
\frac{\left|\Lambda_{n} \cap I\right|-\theta(p)\left|\Lambda_{n}\right|}{r_{n}{ }^{1 / 2}} \Longrightarrow \mathcal{N}(0,1)
$$

with $r_{n}=\operatorname{Var}\left|\Lambda_{n} \cap I\right|$. Our result is a little bit more precise, in the following sense that it gives the asymptotic behavior of $\left(r_{n}\right)_{n \geq 1}: r_{n} \sim \sigma_{p}^{2}\left|\Lambda_{n}\right|$. Thus, independently of [12], we will prove:

Proposition 3.4. Under $\mu_{p}$, we have

$$
\frac{\left|\Lambda_{n} \cap I\right|-\theta(p)\left|\Lambda_{n}\right|}{\left|\Lambda_{n}\right|^{1 / 2}} \Longrightarrow \mathcal{N}\left(0, \sigma_{p}^{2}\right)
$$

where $I$ is the infinite component of $G$.

Proof.

$$
\left|\Lambda_{n} \cap I(\omega)\right|-\theta(p)\left|\Lambda_{n}\right|=\sum_{k \in \Lambda_{n}} f\left(T^{k} \omega\right)
$$

where $T^{k}$ is the translation operator defined by $T^{k}(\omega)=\left(\omega_{n+k}\right)_{n \in \mathbb{Z}^{d}}$ and $f=\mathbb{1}_{\{|C(0)|=+\infty\}}-\theta(p)$. Moreover, $f$ is an increasing function and $\mu^{p}$ satisfies the F.K.G. inequalities. Then $\left.\left(f\left(T^{k} \omega\right)\right)_{k \in \mathbb{Z}^{d}}\right)$ is a stationary random field of square integrable variables satisfying to the F.K.G. inequalities. Therefore, according to Newman [9], the Central Limit Theorem is true if we prove that the quantity

$$
\sum_{k \in \mathbb{Z}^{d}} \operatorname{Cov}\left(f, f \circ T^{k}\right)
$$

is finite.

This way of proving Central Limit Theorems for the density of infinite clusters in percolation models satisfying to the F.K.G. inequalities is not new, because it has already been pointed out by Newman and Schulman $[10,11]$. The new result is that it applies to classical independent bond percolation, i.e. that the series in (7) is convergent.

Next, $\operatorname{Cov}\left(f, f \circ T^{k}\right)=\operatorname{Cov}\left(Y_{0}, Y_{k}\right)$, with $Y_{k}=\mathbb{1}_{\{|C(k)|<+\infty\}}$. Now,

$$
Y_{k}=\sum_{n=0}^{+\infty} F_{n, k}, \text { with } F_{n, k}=\mathbb{1}_{\{|C(k)|=n\}}
$$


Now

$$
\begin{aligned}
\operatorname{Cov}\left(Y_{0}, Y_{k}\right) & =\sum_{n=0}^{+\infty} \sum_{m=0}^{+\infty} \operatorname{Cov}\left(F_{n, 0}, F_{m, k}\right) \\
& =\sum_{n=0}^{+\infty}\left(\operatorname{Cov}\left(F_{n, 0}, F_{n, k}\right)+2 \sum_{m=0}^{n-1} \operatorname{Cov}\left(F_{n, 0}, F_{m, k}\right)\right) \\
& =\sum_{n>\|k\| / 2-1}^{+\infty}\left(\operatorname{Cov}\left(F_{n, 0}, F_{n, k}\right)+2 \sum_{m=0}^{n-1} \operatorname{Cov}\left(F_{n, 0}, F_{m, k}\right)\right) \\
& =\sum_{n>\|k\| / 2-1}^{+\infty} \operatorname{Cov}\left(F_{n, 0}, F_{n, k}+2 \sum_{m=0}^{n-1} F_{m, k}\right)
\end{aligned}
$$

because $F_{n, 0}$ and $F_{m, k}$ are independent as soon as $\|k\| \geq m+n+2$.

Since $F_{n, 0} \geq 0$ and $0 \leq F_{n, k}+2 \sum_{m=0}^{n-1} F_{m, k} \leq 2$, we have

$$
\left|\operatorname{Cov}\left(F_{n, 0}, F_{n, k}+2 \sum_{m=0}^{n-1} F_{m, k}\right)\right| \leq 2 \mathbb{E} F_{n, 0}=2 P(|C(0)|=n) .
$$

Then, $\operatorname{Cov}\left(Y_{0}, Y_{k}\right) \mid \leq \sum_{n>\| k \pi / 2-1} 2 P(|C(0)|=n)$ and

$$
\sum_{k \in \mathbb{Z}^{d}} \operatorname{Cov}\left(f, f \circ T^{k}\right) \leq 2 \sum_{n=1}^{+\infty}\left|\Lambda_{2(n+1)}\right| P(|C(0)|=n) .
$$

Since Kesten and Zhang [8] have proved the existence of $\eta(p)>0$ such that

$$
\forall n \in \mathbb{Z}_{+} \quad P(|C(0)|=n) \leq \exp \left(-\eta(p) n^{(d-1) / d}\right),
$$

it follows that the series converges. Of course, such a sharp estimate is not necessary for our purpose. Estimates derived from Chayes et al. [2], and from Chayes et al. [1] would have been sufficient.

Proof. In this proof, it will be useful to consider $G$ as a random variable. Let $\Omega=\mathcal{S}\left(\mathbb{L}^{d}\right) \times \mathbb{R}^{\mathbb{Z}^{d}}$ and define the probability $\mathbb{P}$ on $\mathcal{B}(\Omega)$ as a skew-product: for measurable $A \times B \in \mathcal{B}\left(\mathcal{S}\left(\mathbb{L}^{d}\right)\right) \times \mathcal{B}\left(\mathbb{R}^{\mathbb{Z}^{d}}\right)$, we have $\mathbb{P}(A \times B)=$ $\int_{A} P^{G, \nu} \mathrm{d} \mu_{p}(G)$. Then, the law of the marginals $G$ and $X$ are $\mathbb{P}_{G}=\mu_{p}$ and $\mathbb{P}_{X}=P^{p, \nu}$.

Rearranging the terms of the sum, we easily obtain

$$
\left.\left(\sum_{x \in \Lambda_{n}} X(x)-((1-\theta(p)) m+\theta(p) Z)\left|\Lambda_{n}\right|\right)\right)=\sum_{x \in \Lambda_{n} \backslash I}(X(x)-m)+(Z-m)\left(\left|I \cap \Lambda_{n}\right|-\left|\Lambda_{n}\right| \theta(p)\right) .
$$

We will now put

$$
\left.Q_{n}=\frac{1}{\left|\Lambda_{n}\right|^{1 / 2}}\left(\sum_{x \in \Lambda_{n}} X(x)-((1-\theta(p)) m+\theta(p) Z)\left|\Lambda_{n}\right|\right)\right)
$$


and define

$$
\forall t \in \mathbb{R} \quad \phi_{n}(t)=\mathbb{E} \exp \left(i Q_{n}\right)
$$

and

$$
\forall t \in \mathbb{R} \quad \phi_{n, z}(t)=\mathbb{E} \exp \left(i Q_{n}\right) \mid\{Z=z\}
$$

As usual, it means that $\mathbb{E}\left(\exp \left(i Q_{n}\right) \mid Z\right)=\phi_{n, z}(Z)$. It is also important to emphasize that the following properties are fulfilled under $\mathbb{P}$ :

- $G$ is independent from $Z$;

- $\left(X_{k} \mathbb{1}_{k \notin I}\right)_{k \in \mathbb{Z}^{d}}$ is independent from $Z$.

Thereby, we have

$$
\phi_{n, z}(t)=\mathbb{E} \exp \left(-\frac{i t}{\left|\Lambda_{n}\right|^{1 / 2}} \sum_{x \in \Lambda_{n} \backslash I}(X(x)-m)+(z-m)\left(\left|I \cap \Lambda_{n}\right|-\left|\Lambda_{n}\right| \theta(p)\right)\right)
$$

Conditioning by $\sigma(G)$ and using the fact that $I$ is $\sigma(G)$-measurable, we get $\phi_{n, z}(t)=\mathbb{E} f_{n}(t,.) g_{n}((z-m) t,$.$) ,$ with

$$
\begin{aligned}
f_{n}(t, \omega) & =\mathbb{E} \exp \left(-\frac{i t}{\left|\Lambda_{n}\right|^{1 / 2}} \sum_{x \in \Lambda_{n} \backslash I}(X(x)-m) \mid \sigma(G)\right) \\
& =\int \exp \left(-\frac{i t}{\left|\Lambda_{n}\right|^{1 / 2}} \sum_{x \in \Lambda_{n} \backslash I(\omega)}(X(x)-m) \mathrm{d} P^{G(\omega), \nu}\right)
\end{aligned}
$$

and

$$
g_{n}(t, \omega)=\exp \left(-\frac{i t}{\left|\Lambda_{n}\right|^{1 / 2}}\left(\left|I(\omega) \cap \Lambda_{n}\right|-\left|\Lambda_{n}\right| \theta(p)\right)\right)
$$

By Theorem 3.1 we have for each $t \in \mathbb{R}$ and $P^{p, \nu}$-almost all $\omega: \lim _{n \rightarrow+\infty} f_{n}(t, \omega)=\exp \left(-\frac{t^{2}}{2} \chi^{f}(p) \sigma^{2}\right)$. Then, by dominated convergence

$$
\lim _{n \rightarrow+\infty} \mathbb{E}\left(f_{n}(t, .)-\exp \left(-\frac{t^{2}}{2} \chi^{f}(p) \sigma^{2}\right)\right) g_{n}((z-m) t, .)=0
$$

Next

$$
\begin{aligned}
\lim _{n \rightarrow+\infty} \mathbb{E} f_{n}(t, .) g_{n}((z-m) t, .) & =\lim _{n \rightarrow+\infty} \exp \left(-\frac{t^{2}}{2} \chi^{f}(p) \sigma^{2}\right) \mathbb{E} g_{n}((z-m) t, .) \\
& =\exp \left(-\frac{t^{2}}{2} \chi^{f}(p) \sigma^{2}\right) \exp \left(-\frac{t^{2}}{2}(z-m)^{2} \sigma_{p}^{2}\right)
\end{aligned}
$$

where the last equality follows from Proposition 3.4. We have just proved that

$$
\lim _{n \rightarrow \infty} \phi_{n, z}(t)=\exp \left(-\frac{t^{2}}{2}\left(\chi^{f}(p) \sigma^{2}+(z-m)^{2} \sigma_{p}^{2}\right)\right) .
$$


Since $\phi_{n}(t)=\int \phi_{n, z}(t) \mathrm{d} \nu(z)$, we get

$$
\begin{aligned}
\lim _{n \rightarrow \infty} \phi_{n}(t) & =\int \exp \left(-\frac{t^{2}}{2}\left(\chi^{f}(p) \sigma^{2}+(z-m)^{2} \sigma_{p}^{2}\right)\right) \mathrm{d} \nu(z) \\
& =\int \exp (i t x) \mathrm{d} \gamma(x) .
\end{aligned}
$$

By the theorem of Levy, it follows that $Q_{n} \Longrightarrow \gamma$.

\subsection{Examples}

\subsection{1. "+/-" valued spin system}

If we choose $\nu=(1-\alpha) \delta_{-1}+\alpha \delta_{1}$ with $\alpha \in(0,1)$, it follows that:

- in the subcritical case $p \in\left(0, p_{c}\right)$, then the limit in the Central Limit Theorem is

$$
\alpha \mathcal{N}\left(0,4 \alpha(1-\alpha) \chi^{f}(p)\right)
$$

- in the supercritical case $p \in\left(p_{c}, 1\right)$, then the limit in the Central Limit Theorem is

$$
\alpha \mathcal{N}\left(0,4 \alpha(1-\alpha) \chi^{f}(p)+4(1-\alpha)^{2} \sigma_{p}^{2}\right)+(1-\alpha) \mathcal{N}\left(0,4 \alpha(1-\alpha) \chi^{f}(p)+4 \alpha^{2} \sigma_{p}^{2}\right) .
$$

\section{Remarks.}

1. For the "+/-" valued spin system in the subcritical case, the annealed Central Limit Theorem can be simply proved without using the quenched one: since $\int \omega_{k} \mathrm{~d} P^{G, \nu}=m$ for each $k$ and each $G$, it follows that the covariance of $X_{0}$ and $X_{k}$ under $P^{p, \nu}$ is

$$
\begin{aligned}
\operatorname{Cov}\left(X_{0}, X_{k}\right) & =\int_{\mathcal{S}\left(\mathbb{L}^{d}\right)}\left(\int\left(\omega_{0}-m\right)\left(\omega_{k}-m\right) \mathrm{d} P^{G, \nu}\right) \mathrm{d} \mu_{p}(G) \\
& =\int \sigma^{2} \mathbb{1}_{\{k \in C(0)\}} \mathrm{d} \mu_{p}(G) \\
& =\sigma^{2} P(k \in C(0)) .
\end{aligned}
$$

Then,

$$
\begin{aligned}
\sum_{k \in \mathbb{Z}^{d}} \operatorname{Cov}\left(X_{0}, X_{k}\right) & =\sum_{k \in \mathbb{Z}^{d}} \int_{\mathcal{S}\left(\mathbb{L}^{d}\right)} \sigma^{2} \mathbb{1}_{\{k \in C(0)\}} \mathrm{d} \mu_{p}(G) \\
& =\sigma^{2} \int_{\mathcal{S}\left(\mathbb{L}^{d}\right)} \sum_{k \in \mathbb{Z}^{d}} \mathbb{1}_{\{k \in C(0)\}} \mathrm{d} \mu_{p}(G) \\
& =\sigma^{2} \int_{\mathcal{S}\left(\mathbb{L}^{d}\right)}|C(0)| \mathrm{d} \mu_{p}(G) \\
& =\sigma^{2} \chi(p),
\end{aligned}
$$

with $\chi(p)=\mathbb{E}|C(0)|=\chi^{f}(p)+\theta(p)(+\infty) . \quad \sum_{k \in \mathbb{Z}^{d}} \operatorname{Cov}\left(X_{0}, X_{k}\right)$ is a convergent series when $p<p_{c}$ and a divergent one else. 
In the subcritical case, the theorem of Newman [9] ensures that the Central Limit is valid as soon as the translation-invariant measure $P^{p, \nu}$ satisfy to the F.K.G. inequalities. Since Häggström and Schramm [7] have proved the F.K.G. inequalities for the "+/-" valued spin system, we get a simple proof for the annealed Central Limit Theorem in this case.

2. When $\alpha=\frac{1}{2}, \gamma$ is a Gaussian measure as well in the subcritical case (the limit is $\mathcal{N}\left(0, \chi^{f}(p)\right)$ ) as in the supercritical case (the limit is $\left.\mathcal{N}\left(0, \chi^{f}(p)+\sigma_{p}^{2}\right)\right)$. It provides an example where there is a classical Central

Limit Theorem whereas the "susceptibility" $\sum_{k \in \mathbb{Z}^{d}} \operatorname{Cov}\left(X_{0}, X_{k}\right)$ is infinite.

It is the "only" case with a Gaussian limit in the supercritical case, as you see from the following remark. 3. If $p \in\left(p_{c}, 1\right)$, then $\gamma$ is Gaussian if and only if there exist $a, b \in \mathbb{R}$ such that $\nu=\frac{1}{2}\left(\delta_{a}+\delta_{b}\right)$.

Proof. Using the characteristic function, it is easy to see that $\gamma$ is Gaussian if and only if $\gamma^{\prime}=\int \mathcal{N}(0$, $\left.(z-m)^{2} \sigma_{p}^{2}\right)(t) \mathrm{d} \nu(z)$ is. Let us define, for $k \in \mathbb{Z}_{+}: m_{k}=\int z^{2 k} \mathrm{~d} \gamma(z)$. We have

$$
\begin{aligned}
m_{k} & =\int \mathcal{N}\left(0,(z-m)^{2} \sigma_{p}^{2}\right)\left(x \mapsto x^{2 k}\right) \mathrm{d} \nu(z) \\
& =\int \frac{(2 k) !}{k ! 2^{k}}(z-m)^{2 k} \mathrm{~d} \nu(z) \\
& =\frac{(2 k) !}{k ! 2^{k}} \int(z-m)^{2 k} \mathrm{~d} \nu(z) .
\end{aligned}
$$

By definition of $\gamma^{\prime}, \gamma^{\prime}$ is a symmetric measure. So if $\gamma^{\prime}$ if Gaussian, it is centered and we have

$$
\forall k \in \mathbb{Z}_{+} \quad m_{k}=\frac{(2 k) !}{k ! 2^{k}} m_{1}^{k}
$$

Then, we have

$$
\forall k \in \mathbb{Z}_{+} \quad \int(z-m)^{2 k} \mathrm{~d} \nu(z)=m_{1}^{k}
$$

If we denote by $\nu^{\prime}$ the image of $\nu$ by $z \mapsto(z-m)^{2}$, we have

$$
\forall k \in \mathbb{Z}_{+} \quad \int_{\mathbb{R}_{+}} z^{k} \mathrm{~d} \nu^{\prime}(z)=m_{1}^{k}
$$

Then, we have

$$
\operatorname{supp} \operatorname{ess} \nu^{\prime}=\lim _{k \rightarrow+\infty}\left(\int_{\mathbb{R}_{+}} z^{k} \mathrm{~d} \nu^{\prime}(z)\right)^{1 / k}=m_{1}=\int_{\mathbb{R}_{+}} z \mathrm{~d} \nu^{\prime}(z)
$$

It follows that for $\nu^{\prime}$-almost all $z, z=\operatorname{supp}$ ess $\nu^{\prime}: \nu^{\prime}$ is a Dirac measure.

Therefore, supp $\nu \subset\left\{m-\sqrt{m_{1}}, m+\sqrt{m_{1}}\right\}$. Since $m=\int z \mathrm{~d} \nu(z)$, we necessary have $\nu\left(m-\sqrt{m_{1}}\right)$ $=\nu\left(m+\sqrt{m_{1}}\right)=\frac{1}{2}$ and then $\nu=\frac{1}{2}\left(\delta_{m-\sqrt{m_{1}}}+\delta_{m+\sqrt{m_{1}}}\right)$. 


\subsubsection{The quenched Gaussian system}

In the case $\nu=\mathcal{N}(0,1)$, Theorem 3.3 takes the following form:

- The subcritical case

If $p \in\left(0, p_{c}\right)$, then

$$
\frac{1}{\left|\Lambda_{n}\right|^{1 / 2}}\left(\sum_{x \in \Lambda_{n}} X(x)\right) \Longrightarrow \mathcal{N}\left(0, \chi^{f}(p) \sigma^{2}\right)
$$

- The supercritical case

If $p \in\left(p_{c}, 1\right)$, then

$$
\frac{1}{\left|\Lambda_{n}\right|^{1 / 2}}\left(\sum_{x \in \Lambda_{n}} X(x)-\theta(p) Z\left|\Lambda_{n}\right|\right) \Longrightarrow \gamma
$$

where $\gamma$ is the image of $\mathcal{N}\left(0, I_{\mathbb{R}^{3}}\right)$ by $(x, y, z) \mapsto\left(\chi^{f}(p)\right)^{1 / 2} x+\sigma_{p} y z$, with

$$
\sigma_{p}^{2}=\sum_{k \in \mathbb{Z}^{d}}\left(P(0 \in I \text { and } k \in I)-\theta(p)^{2}\right),
$$

where $I$ is the infinite component of $G$.

In this case the limit is a Gaussian measure when $p<p_{c}$ whereas it is a Gaussian chaos of order 2 for $p>p_{c}$.

I would like to thank the referees for their carefully reading, specially for suggesting me a simplification in the proof of Theorem 2.1.

\section{REFERENCES}

[1] J.T. Chayes, L. Chayes, G.R. Grimmett, H. Kesten and R.H. Schonmann, The correlation length for the high-density phase of Bernoulli percolation. Ann. Probab. 17 (1989) 1277-1302.

[2] J.T. Chayes, L. Chayes and C.M. Newman, Bernoulli percolation above threshold: An invasion percolation analysis. Ann. Probab. 15 (1987) 1272-1287.

[3] H.-O. Georgii, Spontaneous magnetization of randomly dilute ferromagnets. J. Statist. Phys. 25 (1981) 369-396.

[4] G. Grimmett, Percolation. Springer-Verlag, Berlin, 2nd Edition (1999).

[5] O. Häggström, Positive correlations in the fuzzy Potts model. Ann. Appl. Probab. 9 (1999) 1149-1159.

[6] O. Häggström, R.H. Schonmann and J.E. Steif, The Ising model on diluted graphs and strong amenability. Ann. Probab. 28 (2000) 1111-1137.

[7] O. Häggström, Coloring percolation clusters at random. Stoch. Proc. Appl. (to appear). Also available as preprint http://www.math.chalmers.se/olleh/divide_and_color.ps (2000).

[8] H. Kesten and Yu. Zhang, The probability of a large finite cluster in supercritical Bernoulli percolation. Ann. Probab. 18 (1990) 537-555.

[9] C.M. Newman, Normal fluctuations and the FKG inequalities. Comm. Math. Phys. 74 (1980) 119-128.

[10] C.M. Newman and L.S. Schulman, Infinite clusters in percolation models. J. Statist. Phys. 26 (1981) 613-628.

[11] C.M. Newman and L.S. Schulman, Number and density of percolating clusters. J. Phys. A 14 (1981) 1735-1743.

[12] Yu. Zhang, A martingale approach in the study of percolation clusters on the $\mathbb{Z}^{d}$ lattice. J. Theor. Probab. 14 (2001) $165-187$. 